\title{
New Trends in Raman Spectroscopy: From High-Resolution Geochemistry to Planetary Exploration
}

Olivier Beyssac ${ }^{1}$

\begin{abstract}
This article reviews nonconventional Raman spectroscopy techniques and discusses actual and future applications of these techniques in the Earth and planetary sciences. Time-resolved spectroscopy opens new ways to limit or exploit luminescence effects, whereas techniques based on coherent anti-Stokes Raman scattering or surfaceenhanced Raman spectroscopy allow the Raman signal to be considerably enhanced even down to very high spatial resolutions. In addition, compact portable Raman spectrometers are now routinely used out of the laboratory and are even integrated to two rovers going to Mars in the near future.
\end{abstract}

Keywords: mineralogy, SERS/TERS, time-resolved Raman, luminescence, Mars exploration

\section{INTRODUCTION}

Raman spectroscopy is a valuable, commonly-used, technique in the Earth and planetary sciences. Scientists from this community have been pioneers in using some of the major developments in Raman spectroscopy, such as Raman microprobes, portable Raman instruments, and the technique of Raman hyperspectral mapping. Two obstacles to users are that 1) the Raman signal is inherently weak from most samples studied in the Earth and planetary sciences, and 2) the Raman signal is often parasitized/weakened by other signals, such as luminescence. Therefore, scientists have continuously explored various ways to make the Raman signal stronger and easier to detect. There are alternative ways of exciting/probing the Raman effect, which were imagined back in the 1970s, such as surface- and tip-enhanced Raman spectroscopy (SERS and TERS, respectively), coherent anti-Stokes Raman scattering (CARS), and time-resolved Raman (TRR) spectroscopy. First developed on customized homemade instruments, some of these techniques are now commercially

\footnotetext{
${ }^{1}$ Institut de Minéralogie, de Physique des Matériaux et de Cosmochimie (IMPMC) UMR 7590 Sorbonne Université, CNRS \& MNHN, Campus Jussieu

4 place Jussieu, 75005 Paris, France

E-mail: Olivier.Beyssac@upmc.fr
} 
available and widely used (e.g., SERS, TERS), while others are still under development/application in specific laboratories (TRR, CARS). But these techniques are now slowly emerging in the Earth and planetary sciences. In parallel, progress on the Raman spectrometer hardware, including miniaturization, make the spectrometer a more versatile instrument that can even be deployed out of the laboratory, from museums to deep oceans and as part of Mars rover scientific payloads. This article will provide a glimpse into these new and diverse trends in Raman spectroscopy.

\section{RECONCILING LUMINESCENCE AND RAMAN}

In a Raman experiment, the incident laser beam excites the targeted system into a virtual energy state. Scattering will be essentially elastic, but coupling between the laser's energy and the system's vibrations may trigger the creation and/or the absorption of phonons that produce the Stokes and anti-Stokes Raman effects by inelastic scattering (see Fig. 1 in Pasteris and Beyssac 2020 this issue). When excited by the laser, a sample's structural defects and chemical impurities may contribute to the total signal by emitting a strong background (due to small grain sizes or irregular sample surface) and/or a luminescence signal (due to the presence of organics or other emission centers). Very often, these signals are strong and can overwhelm the weak Raman signal: for instance, luminescence involves electronic transitions and generally complex emission signals. Some emission centers can generate discrete, narrow bands of luminescence in the spectrum, which can be confused with Raman peaks (Lenz et al. 2015). Luminescence is often seen as a limitation for Raman spectroscopy because it generates an intense background that may interfere with the detection of the Raman signal. However, in many cases, the associated luminescence actually carries rich information, so providing a useful signal complementary to Raman (Gaft et al. 2015). Note that "luminescence" is here used as a generic term to describe all kinds of photoluminescence, whatever the mechanism of light emission (e.g., fluorescence, luminescence, or phosphorescence) (see Gaft et al. 2015 for further details).

\section{Tuning Laser Wavelength}

Many natural samples exhibit an inherent background, which often overwhelms the Raman signal. This may be due to rough surfaces or small grain sizes on the same order of magnitude as the laser wavelength. This is not recommended for conventional Raman analysis because the laser will interact with the surface rather than the volume of the particles and/or may even be diffracted by them for sizes 
smaller than the laser wavelength. Laser light will be scattered within samples having a small grain size and be returned to the spectrometer. Although most current Raman instruments have a single-stage monochromator that yields high luminosity, there is poor rejection efficiency of parasitic light responsible for the background. Another possible issue is luminescence, which is due to the radiative relaxation of electronic excitation in molecules (see Fig. 2 in Pasteris and Beyssac 2020 this issue). By itself, luminescence is a spectroscopic technique used in various fields of the Earth sciences (Gaft et al. 2015) and in disciplines such as biomedicine, with its use of fluorescence spectroscopy (Lakowicz 2006). Each emission center has a specific absorption spectrum of incident light. After photons are absorbed, the system relaxes and energy is released as an emission spectrum specific to the element of interest in the host matrix. This emission spectrum is the signal that may be observed in the spectral window monitored for Raman spectroscopy. Importantly, the Raman shift corresponds to an energy absorbed and released by the sample, specifically referenced to the energy of the laser excitation. Thus, as the excitation wavelength/energy of the laser is varied, Raman peaks move in the light spectrum as viewed with respect to absolute wavenumber coordinates. In contrast, the positions of luminescence emission bands are constant with respect to absolute wavenumbers, whatever the exciting laser wavelength: they may or may not appear in the spectral window considered for Raman spectroscopy (FIG. 1).

Most commercial instruments offer several excitation wavelengths, from deep ultraviolet (UV) to near-infrared (IR). Basically, the intensity of the Raman signal is proportional to $1 / \lambda^{4}$, where $\lambda$ is the wavelength of the incident laser beam, but various constraints have to be considered when selecting a laser wavelength for Raman spectroscopy (Dubessy et al. 2012; Pasteris and Beyssac 2020 this issue). FIGURE 1 depicts the laser-dependent Raman spectral windows and scattering efficiency from the deep UV to near-IR, as well as the region where mostluminescence is detected (visible to near-IR). Ultraviolet light is highly energetic and generally efficiently absorbed. Due to strong coupling, as well as scattering, the Raman signal is actually very strong in the UV. Luminescence is also likely excited in the sample, but it does not overlap and, therefore, does not interfere with the Raman signal. This is because the (Stokes) Raman spectral window is compressed in absolute wavelength in the UV and is shifted to lower wavelengths with respect to luminescence (FIG. 1). The drawback is that UV light requires special optics and detectors, as well as avoidance of the effects of UV light (e.g., photo-oxidation) on the sample target. A specific use of UV Raman is resonance Raman: by tuning the laser wavelength (e.g., 
with a dye laser), it may be possible to match its energy with that of an absorption band of the sample, corresponding to an electronic transition (see FIG. 2 in Pasteris and Beyssac 2020 this issue). This will specifically enhance (by a theoretical factor of up to $10^{6}$ ) the intensity of the Raman peak associated with the electronic transition probed. This technique works well for studying organic compounds (Steele et al. 2020 this issue) but is rarely used in the Earth sciences for minerals (Tarcea et al. 2007).

On the other side of the visible light spectrum, near-IR is less strongly absorbed than UV. Therefore, the excitation of luminescence is weaker, and the Raman spectral window is shifted to yet longer wavelengths, making the luminescence not visible. But, due to the $1 / \lambda^{4}$ scattering effect, near-IR light does not efficiently excite the Raman effect. In this region, the Raman signal is actually weak and most easily detected for targets with high Raman scattering cross-sections at this wavelength (e.g., carbonates, phosphates, sulfates). However, there is a specific signal-handling use of near-IR excitation called Fourier transform (FT) Raman, which is generally implemented with a 1,064-nm laser and a sensitive single-element, near-IR detector (e.g., InGaAs): this is because a charge-coupled device (CCD) has a very low detection efficiency at this long wavelength. Whereas a dispersive Raman system uses a grating to disperse light according to wavelength, an interferometer is used for FT Raman. It converts the Raman signal into an interferogram and allows the collection of the entire Raman spectrum simultaneously on the detector (Parker 1994). The drawbacks are that the Raman signal is inherently very weak, the spatial resolution is low compared to that of visible light, and the spectral domain is restricted by the sample size due to the diffraction limit of the long excitation wavelength. Therefore, this technique is rarely used in the Earth sciences. It is used in polymer, medical, or forensic sciences, where molecules are highly luminescent and have high Raman scattering cross-sections.

For all these reasons, a good compromise in the Earth sciences is generally to use visible light to excite the sample, typically in the range 450-650 $\mathrm{nm}$. These wavelengths are easy to handle instrumentally, they enable good spatial resolution, and they efficiently excite the Raman effect (Dubessy et al. 2012).

\section{Time-Resolved Spectroscopy}

Another way to separate the Raman signal from luminescence is to play with the fact that these two processes have very differentlifetimes. Thus, we can use time-resolved spectroscopy. "Lifetime" corresponds to the time spent by an emission center 
(sometimes called an activator) in the excited state before it returns to the ground state and emits a photon. A lifetime includes both absorption (nearly immediate) and relaxation (variable duration). Relaxation from virtual vibrationallevels is immediate for Raman, typically in the range of $10^{-14}$ seconds. On the other hand, relaxation from excited electronic levels is more sluggish, and the lifetime of luminescence covers a wide time range from the nanosecond in organic fluorescence (Lakowicz 2006) to milliseconds or even seconds in minerals (e.g., $\mathrm{Cr}^{3+}$ or $\mathrm{REE}^{3+}$ ) (Gaft et al. 2015).

Time-resolved spectroscopy uses a pulsed excitation signal synchronized with a gated detector. The main idea is that the Raman signal is excited immediately by and will last only during the excitation pulse, while the luminescence will be excited during the pulse but will continue to decay after the pulse. Using a short(-time) gate that matches the laser pulse in the time domain and that is perfectly synchronized with it, allows rejection of most of the luminescence signal and maximum collection of the Raman signal (FIG. 2). Time-resolved spectroscopy further allows collection of only the luminescence signal, by opening the detector gate just after the laser pulse. The Raman signal is by then absent, because its excitation has relaxed, while the luminescence is still present. Using advanced instruments with perfect synchronization, it is possible to explore luminescence with various decay periods by using smart combinations of delays (moment the detector gate opens with respect to the laser pulse) and open-gate duration. Lastly, this technique allows proper characterization of the time decay of luminescence. A post pulse detector gate with appropriate opening duration is set to various delay times (gate opening after laser pulse), and the integrated intensity of the signal is plotted versus the delay time. These data give further information on the nature of the electronic transition and, more practically, on the identification of the emission center (Lakowicz 2006; Gaft et al. 2015).

Experimentally, instruments for time-resolved spectroscopy are mostly customized homemade instruments, although some manufacturers now propose time-resolved instruments for specific applications. In most cases, these setups use an intensified charge-coupled device (ICCD) with a photocathode for the gating (down to the nanosecond) and a photon intensifier to enhance the signal on the CCD. Alternatives are single-photon avalanche diode detectors (Blacksberg et al. 2016) or a picosecond Kerr gating (Matousek et al. 2002). For the excitation, pulsed lasers are used with pulse durations generally in the nanosecond range, which is similar to that achievable for ICCD gating. Choice of laser wavelengths obeys the same constraints as for 
continuous-wave Raman (see above). In the Earth and planetary sciences, several instrumental setups have been developed for standoff remote analysis by collimating the laser on the sample surface and collecting light through a telescope. The motivation here is to refine instruments for the field, including planetary exploration (see below), and to reject the daylight contribution by time-resolution. Some very nice results have been obtained with these systems, such as retrieving clean Raman spectra from highly luminescent minerals or performing Raman spectroscopy at distances of up to several tens of meters on large crystals/rocks (Sharma et al. 2009).

\section{HIGH-RESOLUTION GEOCHEMISTRY WITH COHERENT OR ENHANCED RAMAN SPECTROSCOPY}

New scientific questions drive the need to observe at ever higher spatial resolution and/or detect ever smaller amounts of a given molecule. The Raman signal is, however, inherently weak, especially for individual components in complex targets, which may be solid, fluid, mineral, or organic. Therefore, various strategies have been deployed to make the Raman signal stronger for spectroscopy and for imaging.

\section{Coherent Raman Spectroscopy}

Coherent Raman scattering includes an ensemble of techniques based on a moleculespecific, nonlinear, optical interaction sensitive to Raman vibrational resonances. These techniques generally rely on the interaction of two laser beams: a pumped laser and a Stokes laser, for which their frequency difference matches a resonant vibrational Raman frequency of the molecule of interest. The techniques mainly differ in the detection of the Raman signal: the homodyne technique like coherent antiStokes Raman scattering (CARS), which directly detects the anti-Stokes signal, versus the heterodyne technique, such as stimulated Raman scattering (SRS), which detects the transferred modulation of either the pumped or the Stokes beam (Houle et al. 2017). These techniques are well developed in biomedicalscience to characterize, for example, lipid-rich structures using the $\mathrm{C}-\mathrm{H}$ stretching vibration. Although such techniques require complex experimental setups and advanced expertise in spectroscopy, they have been applied successfully in the Earth sciences. An outstanding example is the characterization and 3-D imaging of fluid inclusions by combining CARS with a laser scanning confocal microscope (Burruss et al. 2012). In this case, the strong resonant character of molecules like $\mathrm{CH}_{4}, \mathrm{CO}_{2}, \mathrm{H}_{2} \mathrm{O}$, or even hydrocarbons is used to generate 3-D images of the inclusions. The high spatial resolution and superb quality of these images open new doors for investigating not only the volumetric relations of the gases in the inclusions but also the microtextural 
features of the inclusions within the microfractures of the host mineral. Other applications of CARS for the microtextural imaging of carbonates in microfossils or sulfate inclusions in a calcite crystal are presented and discussed by Houle et al. (2017).

\section{SERS and TERS}

Surface-enhanced Raman spectroscopy (SERS) offers perhaps the most spectacular amplification of the Raman signal. Although the first observations of the physical effects involved in SERS were made in the 1970s, the technique really took off during the early $21^{\text {st }}$ century. Many fields now routinely use SERS, from material sciences to biomedicine (Cialla et al. 2012), but still only rarely in the Earth sciences. Basically, SERS analyzes molecules adsorbed onto a specifically prepared metal surface (FIG. 3). This metal surface should have plasmonic properties: at the interface between the metal and a dielectric material (something nonconductive, such as air), the incident light from the laser interacts with the free electrons of the metal to create a resonant interaction, which produces oscillations of the electrons. At the metal's surface this triggers the formation of electron density waves called plasmons. Electromagnetic enhancement of the Raman signal results from the amplification of the light by the excitation of localized plasmon resonances, which theoretically can reach factors of $10^{10}-10^{11}$, but more practically $10^{6}$, depending on the sample and the supporting plasmonic material. There is also a chemical enhancement, not yet completely understood, but probably due to charge transfer mechanisms in which the laser excitation wavelength is resonant with the electronic states of the metal-molecule charge transfer. Theoretically, chemical enhancement can reach up to a factor of $10^{3}$. A complete description of SERS is given by Aroca (2006).

Practically, SERS can be done with any conventional Raman microspectrometer that has good confocality and a high collection efficiency: the enhancement is localized within the very close vicinity of the substrate surface and rapidly decreases outward. The key aspect is the choice of the plasmonic substrate, which should have a peak of plasmon resonance in accordance with the laser wavelength. Typically, metals like $\mathrm{Cu}, \mathrm{Au}$, or $\mathrm{Ag}$ are used as substrates with laser wavelengths in most of the visible and near-IR region. In addition, these metals yield good interaction with many adsorbed molecules. Considerable work has been done to look for new SERS substrates (e.g., graphene, semiconductors, quantum dots) and/or to optimize the configuration of the substrate (shape, surface properties) to maximize the signal enhancement. 
Tip-enhanced Raman spectroscopy (TERS) is, in a way, derived from SERS, but the electromagnetic field enhancement is restricted to a sharp metallic tip irradiated by the laser light (FIG. 3). The sample is moved very close to the metal tip, which creates a localized region of SERS enhancement. The main advantage is the excellent and accurate spatial resolution reached by TERS, which allows structural and chemical characterization at the nanometer scale. Practically, TERS is performed by combining a Raman spectrometer with a scanning tunneling microscope (STM) or an atomic force microscope (AFM). Tip-enhanced Raman spectroscopy holds fantastic potential for analyzing and mapping at the nanometer scale, although further knowledge is required for precise estimation of the region of enhancement to properly assess the intrinsic resolution of the method and its real enhancement efficiency.

The SERS/TERS methods are now routinely and successfully used across a range of scientific disciplines-material sciences, biochemistry, biosensing, catalysis, electrochemistry, and forensic sciences-for which characterizing small amounts (almost at the single-molecule level) of organic compounds is critical. There are many fewer applications in the Earth sciences, although SERS/TERS may be used as an indicator of surface properties, thereby making it relevant for some specific mineral investigations or for the detection of organic molecules on mineral surfaces (Mernagh et al. 2018). In the environmental sciences, SERS has been used to detect and characterize organic or inorganic aqueous or airborne contaminants, as well as to detect microbes or nanomaterials in environmental samples (Halvorson et al. 2010).

\section{TAKING RAMAN OUT OF THE LABORATORY}

Usually, a sample is carried to the laboratory where it is prepared (e.g., thin section, crushing) and then analyzed with instruments in an optimized environment. But sometimes it may be preferable to take a Raman instrument out of the lab to analyze a sample in the field. The reasons are multiple and diverse, from exploration geology in the field to analysis of priceless art or gem artifacts, which cannot be taken out of museum cabinets: this latter enables the study of nontransportable, fragile, and also large archeological objects, such as statues. There are not so many analytical techniques that are nondestructive and that can be deployed out of the lab routinely. Raman spectroscopy is one of the few, another being the complementary technique of X-ray fluorescence, which provides chemical information. Taking instruments out of the lab raises numerous challenges in terms of portability, mechanical reliability, resistance to environment, and energy autonomy. Fortunately, these have been progressively overcome for Raman spectroscopy. 
There are two main approaches in terms of instrumentation: 1) moving and adapting a conventional, but mobile, laboratory instrument; or 2) using a dedicated portable instrument. The first approach is possible with most commercial instruments but is limited to in-room experiments. Microscopes may still be used, but generally an optical fiber is used to transport the excitation laser and so enable Raman collection directly on the sample without moving it. At the end of the optical fiber, various kinds of optical heads may be adapted, such as microscope objectives or lenses with longer focal distances. Such an approach is used successfully in museums for studying pigments in paintings or analyzing gems on precious artifacts (Vandenabeele et al. 2007; Bersani et al. 2016). Such a configuration is also used on large instrumental facilities, such as a synchrotron, where Raman spectroscopy may be used to complement other techniques based on X-rays. Portable Raman instruments have existed commercially for nearly two decades, and there is now a large number of offerings with various collection configurations and excitation wavelengths (Jehlička et al. 2017). In recent years, transmission spectrometers (those using transmissive gratings), gained in popularity, because they offer good spectral resolution and make easier the design of compact instruments.

With instruments going to Mars (see below), one specific application has been the design of Raman spectrometers for deep-ocean exploration (Pasteris et al. 2004). Such instruments have been deployed thanks to remotely operated vehicles providing energy, data flow, and accurate positioning on targets of interest. These deep-ocean instruments are used to identify, and even quantify, the solutes present in seawater and to detect and characterize complex gas hydrates present in the deepocean. Future applications may include biologicalinvestigations based on Raman and luminescence signals. A first attempt to characterize the pigments present in a red fish was successful, although the story does not tell how the fish felt after being shot by the green laser (Zhang et al. 2011).

\section{RAMAN IS GOING TO MARS!}

Raman spectrometers have been selected for the scientific payload of two rovers going to Mars in the near future. Here is further recognition for the technique, which is long overdue on Mars: a scheduled Raman instrument was removed from the scientific payload of the now legendary Mars Exploration rovers Spirit and Opportunity in 2004. The two next missions, NASA's Perseverance rover and ESA/Roscosmos Exomars, have the same main scientific objective, which is to identify any trace evidence of ancient life on the surface of this planet. Raman spectroscopy is 
expected to play a crucial role in the detection and characterization of possible organics, but also to characterize minerals that may have been involved in biomineralization and fossilization processes, such as carbonates, phosphates, and some oxides (see Steele et al. 2020 this issue). More generally, Raman instruments are expected to provide structural and mineralogical information about Martian materials. Interestingly, three instruments will be deployed with very different strategies: the SuperCam and SHERLOC (Scanning Habitable Environments with Raman and Luminescence for Organics and Chemicals) instruments onboard the Perseverance rover and the RLS (Raman LaserSpectrometer) instrument onboard the Exomars rover (named Rosalind Franklin). Of course, these instruments represent an advanced engineering challenge in terms of compactness (in general they weigh only a few kilograms) and their resistance to extreme conditions (e.g., mechanical shocks, large thermal variations, and dust).

The Rosalind Franklin rover will carry a complete suite of scientific instruments to fully characterize the samples in situ. The selected landing site is Oxia Planum, a Noachian (Archean equivalent) clay-rich, vast, lowland plain near the equator. This rover is supposed to drill down to two meters through the Martian rocks and to crush the samples for advanced characterization. The objective is to characterize pristine rocks (via drilling) that were not affected by the intense UV and cosmogenic irradiation that affects the upper centimeters of the Martian surface, because such irradiation can be detrimental for organics. The RLS instrument is part of the Pasteur payload [also including the micrOmega infrared spectrometer and MOMA (Mars organic molecule analyzer) mass-spectrometer instruments] located in the body of the rover. Protected from the external environment, the RLS will operate in the dark to analyze powders carried by a carousel after the rocks are crushed. The instrument comprises a continuous laser (Nd:yttrium-aluminum-garnet at $532 \mathrm{~nm}$ ), an optical head focusing the laser to a $50-\mu \mathrm{m}$ spot size and that collects the signal, a compact transmission spectrometer, and a CCD detector. The instrument should be able to characterize most minerals, including hydrated ones, as well as organics (Rull et al. 2017).

The Perseverance rover will carry a full suite of scientific instruments for in situ characterization of the Martian rocks. This rover is also equipped with a cache system and is supposed to assemble 20 samples that will be collected by a future Mars mission and return these samples to Earth (Williford et al. 2017). Mars2020 will land in the Jezero Crater, which is a Noachian crater basin at the western edge of the Isidis 
Basin. It has been characterized as fluvial/deltaic sediment deposited into a circumneutral/low-salinity (i.e., potentially habitable) paleolake. The SHERLOC instrument is located on the arm turret of the rover and is associated with the PIXL (planetary instrument for X-ray lithochemistry) instrument, which uses X-ray fluorescence for chemical characterization to complement the information provided by SHERLOC. These two instruments will work close to the sample after mechanical abrasion of the target to remove any dust and alteration layers (e.g., varnishes). The SHERLOC instrument is a deep-UV (laser excitation at $248.6 \mathrm{~nm}$ ) Raman spectrometer optimized to detect, analyze, and map the distribution of organics in the targeted rock (Abbey et al. 2017). The poor penetration of the UV laser limits excitation to only the sample's surface, but low irradiance of the sample can be maintained because of the (1) high efficiency of Raman scattering in the UV (FIG. 1), and (2) possible resonance enhancement effects for organics with aromatic or conjugated bonds. The key advantage here is that the Raman spectral window is free of luminescence. The SHERLOC instrument will be combined with a high-resolution context imager called Watson.

The second Raman instrument onboard Perseverance is SuperCam (FIG. 4). This is a multifunctional spectroscopy instrument, a "Swiss Army-knife instrument", built by a USA-France-Spain consortium. The design is largely inherited from that of the ChemCam instrument onboard the Curiosity rover, which has been successfully analyzing the surface of Mars since 2012. SuperCam will remotely analyze the surface using laser-induced breakdown spectroscopy (LIBS), time-resolved Raman and luminescence spectroscopy, and visible-infrared (VISIR) spectroscopy. A color remote micro-imager (RMI) will provide context images and textural detail, and a microphone will record the LIBS plasma shock waves and the sounds of Mars (Wiens et al. 2016). For the Raman analyses, SuperCam faces two main technical challenges (compared to laboratory instruments): 1) remote analysis in daylight and timeresolved spectroscopy; 2) it will have to work on Mars. To do this, SuperCam combines a unit on top of the rover mast that can be operated in all directions and that includes a telescope and a pulsed laser (nanosecond pulses at $532 \mathrm{~nm}$ ). SuperCam has a body unit that includes a transmission spectrometer and an ICCD in the rover body, and the two instruments are connected by an optical fiber and cables (Wiens et al. 2016). Time-resolution down to $100 \mathrm{~ns}$ on the ICCD allows for rejection of the ambient light continuously entering the telescope. It avoids unwanted luminescence, but can also exploit luminescence for the possible detection of trace elements (such as the rare earth elements) not achievable by LIBS. SuperCam is 
expected to be the "eye" of the rover by shooting rocks at distances of up to several meters to provide quantitative chemical (LIBS) and qualitative mineralogical(Raman and VISIR) information. Owing to its relative simplicity and speed of operation, SuperCam is supposed to guide the rover's displacement, to provide key information for the operation of other scientific instruments, and to document the geological context for selection of the sites to be sampled for Mars sample return.

\section{SUMMARY}

Conventional Raman spectroscopy is now routinely used in Earth science institutions (e.g., Chou and Wang 2017) and new developments are progressively emerging in this field to enhance the inherently weak Raman signal and/or to work at higher spatial resolutions. Raman mapping has also undergone a revolution and now can be done easily and it can complement other imaging techniques based on X-ray fluorescence (Nasdala et al. 2012). "Outdoor" applications, including Mars exploration, clearly open new avenues for in situ mineralogy and geochemistry but face numerous complex technological challenges to capture the rich, yet subtle and weak, Raman signal.

\section{ACKNOWLEDGMENTS}

Thanks are due to Terry Mernagh, Shiv Sharma, and Dominique De Ligny for insightful reviews, to Roger Wiens for reading a preliminary version of the manuscript and to Michel Gauthier for numerous spectroscopy discussions. Sincere thanks to guest editor Jill Pasteris for extremely patient and constructive handling of the manuscript. Funding from CNRS and Sorbonne Université is acknowledged.

\section{REFERENCES}

Abbey WJ and 10 coauthors (2017) Deep UV Raman spectroscopy for planetary exploration: the search for in situ organics. Icarus 290: 201-214

Aroca, R (2006) Surface Enhanced Vibrational Spectroscopy. Wiley, Hoboken, New Jersey, USA, $260 \mathrm{pp}$

Bersani D, Conti C, Matousek P, Pozzi F, Vandenabeele P (2016) Methodological evolutions of Raman spectroscopy in art and archaeology. Analytical Methods 8: 8395-8409

Blacksberg J, Alerstam E, Maruyama Y, Cochrane CJ, Rossman GR (2016) Miniaturized time-resolved Raman spectrometer for planetary science based on a fast single photon avalanche diode detector array. Applied Optics 55: 739-748 
Burruss RC, Slepkov AD, Pegoraro AF, Stolow A (2012) Unraveling the complexity of deep gas accumulations with three-dimensional multimodal CARS microscopy. Geology 40: 1063-1066

Chou I-M, Wang A, (2017) Application of laser Raman micro-analyses to Earth and planetary materials. Journal of Asian Earth Sciences 145: 309-333

Cialla D and 6 coauthors (2012) Surface-enhanced Raman spectroscopy (SERS): progress and trends. Analytical and Bioanalytical Chemistry 403: 27-54

Dubessy J, Caumon M-C, Rull F, Sharma S (2012) Instrumentation in Raman spectroscopy: elementary theory and practice. In: Dubessy J, Caumon M-C, Rull F (eds) Raman Spectroscopy Applied to Earth Sciences and Cultural Heritage. European Mineralogical Union Notes in Mineralogy 12, European Mineralogical Union and Mineralogical Society of Great Britain \& Ireland, London, pp 83-172

Fau A and 15 coauthors (2019) Pulsed laser-induced heating of mineral phases: implications for laser-induced breakdown spectroscopy combined with Raman spectroscopy. Spectrochimica Acta Part B: Atomic Spectroscopy 160, doi: 10.1016/j.sab.2019.105687

Gaft M, Reisfeld M, Panczer M (2015) Modern Luminescence Spectroscopy of Minerals and Materials. $2^{\text {nd }}$ Edition. Springer International Publishing, Switzerland, $606 \mathrm{pp}$

Halvorson RA, Vikesland PJ (2010) Surface-enhanced Raman spectroscopy (SERS) for environmental analyses. Environmental Science \& Technology 44: 77497755

Houle M-A and 5 coauthors (2017) Rapid 3D chemical-specific imaging of minerals using stimulated Raman scattering (SRS) microscopy. Journal of Raman Spectroscopy 48: 726-735

Jehlička J, Culka A, Bersani D, Vandenabeele P (2017) Comparison of seven portable Raman spectrometers: beryl as a case study. Journal of Raman Spectroscopy 48: $1289-1299$

Lakowicz JR (2006) Principles of Fluorescence Spectroscopy. $3^{\text {rd }}$ Edition. SpringerVerlag US, $954 \mathrm{pp}$

Lenz C and 5 coauthors (2015) Laser-induced REE ${ }^{3+}$ photoluminescence of selected accessory minerals - an "advantageous artefact" in Raman spectroscopy. Chemical Geology 415: 1-16

Matousek P, Towrie M, Parker AW (2002) Fluorescence background suppression in Raman spectroscopy using combined Kerr gated and shifted excitation Raman difference techniques. Journal of Raman Spectroscopy 33: 238-242

Mernagh TP, King PL, McMillan PF, Berger JA, Dalby KN (2018) Using infrared and Raman spectroscopy to analyze gas-solid reactions. Reviews in Mineralogy and Geochemistry 84,177-228 
Nasdala L, Beyssac O, Schopf JW, Bleisteiner B (2012) Application of Raman-based images in the Earth sciences. In: Zoubir A (ed) Raman Imaging. Springer Nature, Cham, Switzerland, pp 145-187

Panczer G and 5 coauthors (2012) Raman and fluorescence. In: Dubessy J, Caumon M-C, Rull F (eds) Raman Spectroscopy Applied to Earth Sciences and Cultural Heritage. European Mineralogical Union Notes in Mineralogy 12, European Mineralogical Union and Mineralogical Society of Great Britain \& Ireland, London, pp 61-82

Parker SF (1994) A review of the theory of Fourier-transform Raman spectroscopy. Spectrochimica Acta Part A: Molecular Spectroscopy 50: 1841-1856

Pasteris JD and 6 coauthors (2004) Raman spectroscopy in the deep ocean: successes and challenges. Applied Spectroscopy 58: 195-208

Pasteris JD, Beyssac 0 (2020) Welcome to Raman spectroscopy: successes, challenges, and pitfalls. Elements 16: XXX-XXX

Rull F and 25 co-authors (2017) The Raman Laser Spectrometer for the ExoMars Rover mission to Mars. Astrobiology 17: 627-654

Sharma SK, Misra AK, Lucey PG, Lentz RCF (2009) A combined remote Raman and LIBS instrument for characterizing minerals with $532 \mathrm{~nm}$ laser excitation. Spectrochimica Acta Part A: Molecular and Biomolecular Spectroscopy 73: 468-476

Steele A, Fries M, Pasteris J (2020) Geoscience meets biology: Raman spectroscopy in geobiology and biomineralization. Elements 16: XXX-XXX

Tarcea N and 7 coauthors (2007) UV Raman spectroscopy-a technique for biological and mineralogical in situ planetary studies. Spectrochimica Acta Part A: Molecular and Biomolecular Spectroscopy 68: 1029-1035

Vandenabeele P, Edwards HGM, Moens L (2007) A decade of Raman spectroscopy in art and archaeology. Chemical Reviews 107: 675-686

Wiens RC, Maurice S, Rull F (2016) The SuperCam remote sensing instrument suite for the Mars 2020 Rover: a preview. Spectroscopy 32: 50-55

Williford KH and 15 coauthors (2017) The NASA Mars 2020 Rover Mission and the search for extraterrestrial life. In Cabrol NA, Grin EA (eds) From Habitability to Life on Mars. Elsevier, Amsterdam, pp 275-308

Zhang X, Kirkwood WJ, Walz PM, Peltzer ET, Brewer PG (2012) A review of advances in deep-ocean Raman spectroscopy. Applied Spectroscopy 66: 237-249 


\section{FIGURE CAPTIONS}

FIGURE 1 Raman spectral windows for excitation at various laser wavelengths. For each laser, the dark bar on the left side of each color-shaded box represents the laser wavelength; two schematic Raman peaks at 500 and 1,000 $\Delta \mathrm{cm}^{-1}$ are depicted within each box to show a 2,000 $\mathrm{cm}^{-1}$ spectral window. Also shown are the general range of luminescence emissions ("luminescence/organic fluorescence" dark grey bar in middle of figure) and the relative efficiency of Raman scattering as a function of wavelength (red curve in upper part of figure). Abbreviation: a.u. = arbitrary units; IR = infrared. Equation in top grey box indicates how the intensity of the Raman shift is related to the exciting light wavelength. ModifiEd AFTER PANCZER ET AL. (2012).

FIGURE 2 Time-resolved Raman and luminescence spectroscopy of a zircon grain. (LEFT) Schematic illustrations of the synchronization between the laser pulse and the intensified charge-coupled device (ICCD) gate to catch pulse-synchronous Ramanonly spectra (green; middle graph), or slightly post-pulse luminescence only (dashed pale blue line for long lifetime; dashed dark blue line for short lifetime; lower graph), or both (upper graph). (RIGHT) Total continuous-wave (CW) excitation Raman spectra, Raman spectra (green curve, peaks highlighted in yellow), and luminescence (here, due to $\mathrm{REE}^{3+}$ in the zircon sample) spectra (red curve).

FIGURE 3 (A) Schematic illustration of the surfaced-enhanced Raman spectroscopy (SERS) technique; the region of enhanced Raman is located at the surface of metal nanoparticles. (B) Schematic illustration of the tip-enhanced Raman spectroscopy (TERS) technique; the region of enhanced Raman is located at the edge of the metal tip.

FIGURE 4 Schematic diagram of the SuperCam instrument on board the NASA Mars 2020 mission. (UPPER) The mast unit, containing the laser, the telescope, the remote micro-imager (RMI) and the infrared (IR) spectrometer. (LOWER) The body unit is connected to the mast unit by an optical fiber and contains the ultraviolet (UV), violet (Vio) and transmission (trans.) spectrometers and detectors [demux = demultiplexer]. The instrument has a complete set of calibration targets for each technique, including a diamond for Raman. MODIFIED AFTER WIENS ET AL. (2016). 


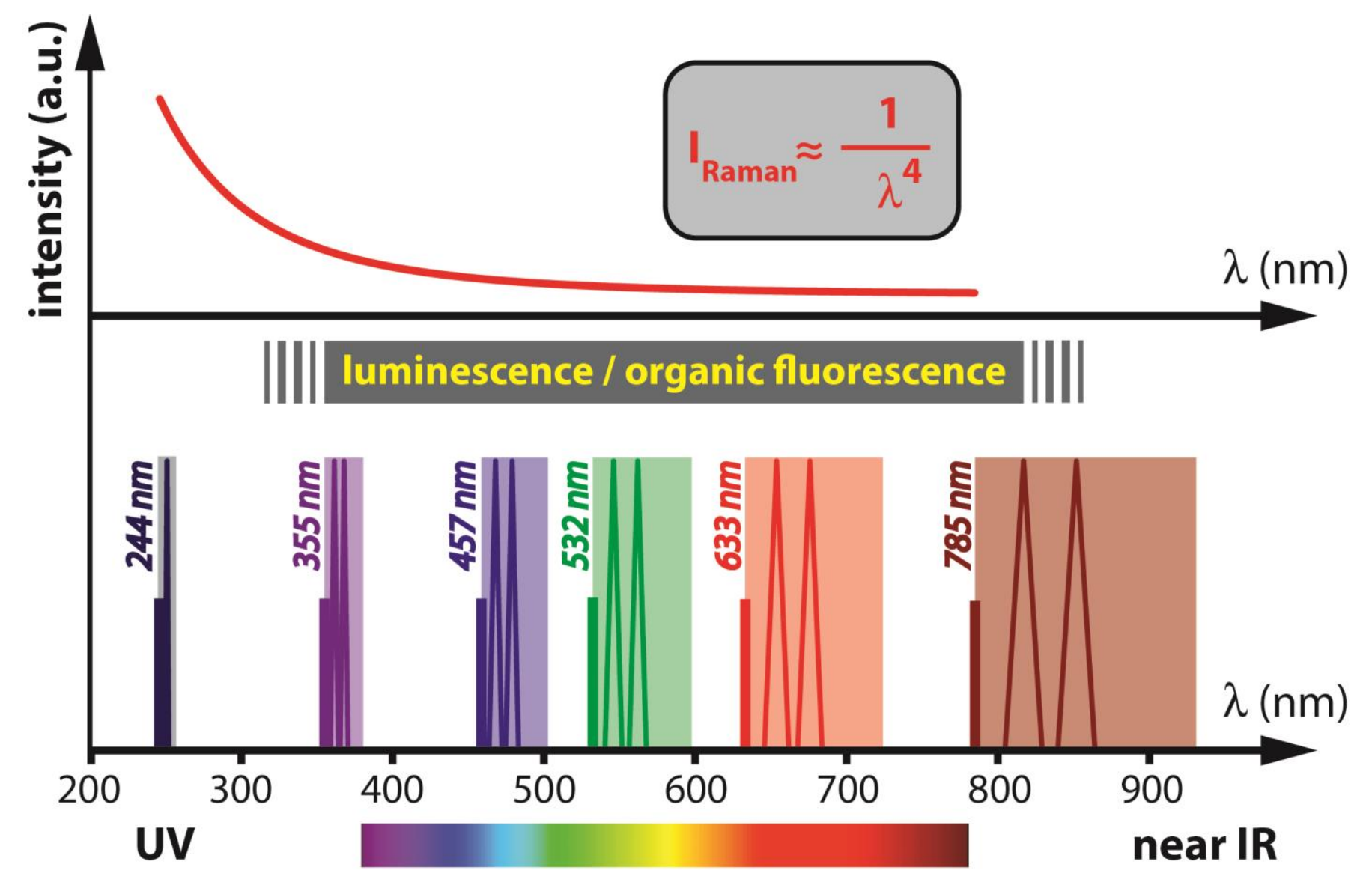

Figure 1 


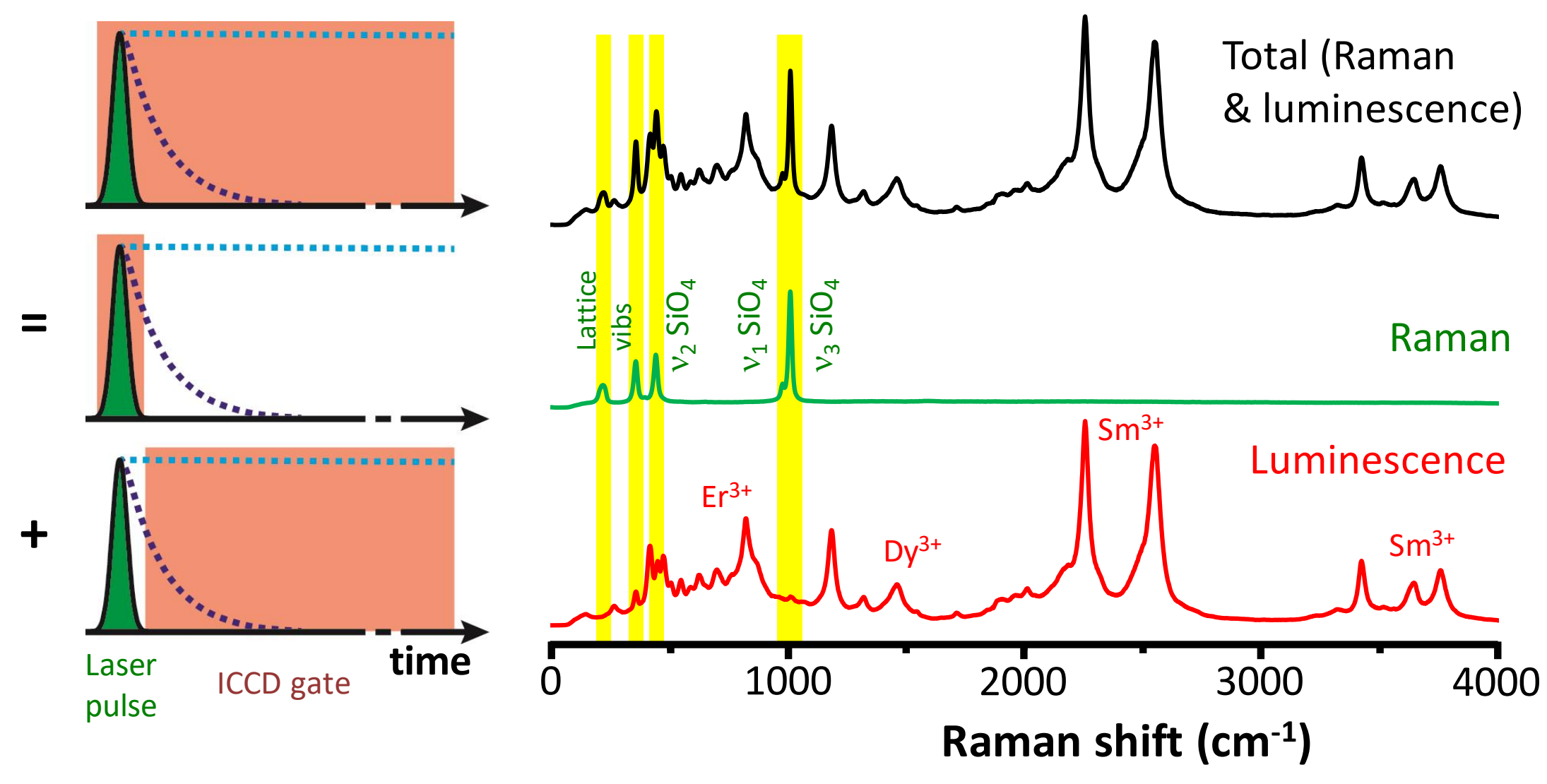

Figure 2 

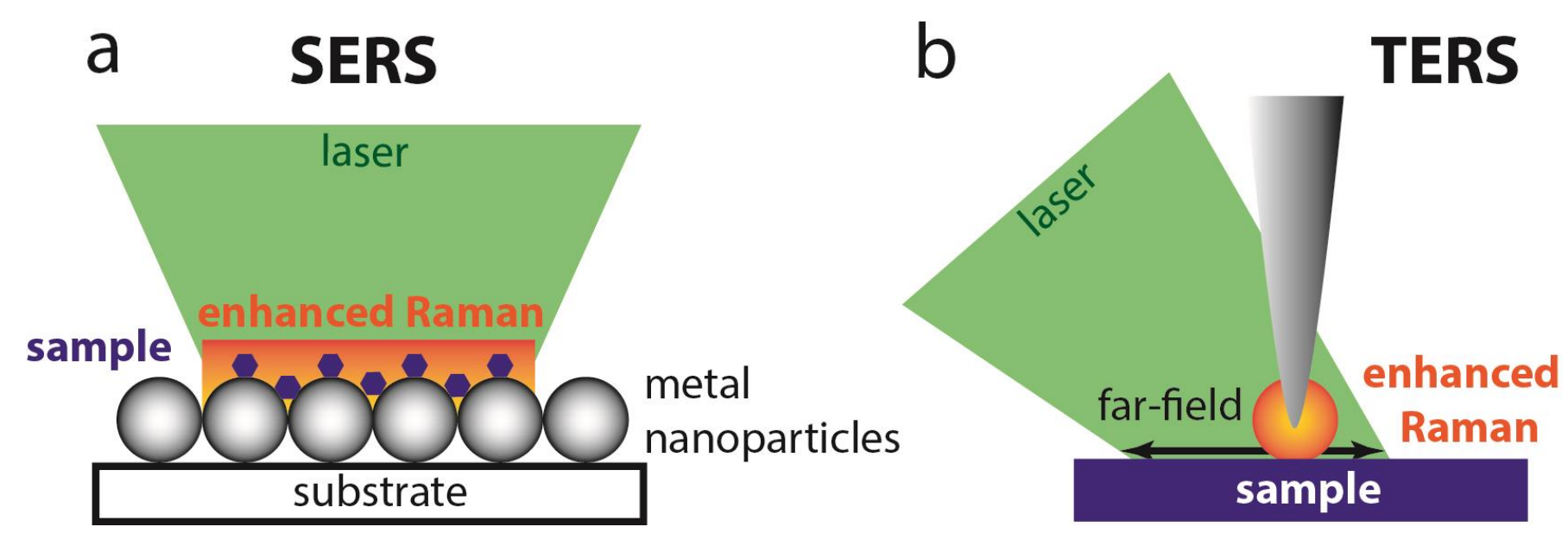

Figure 3 


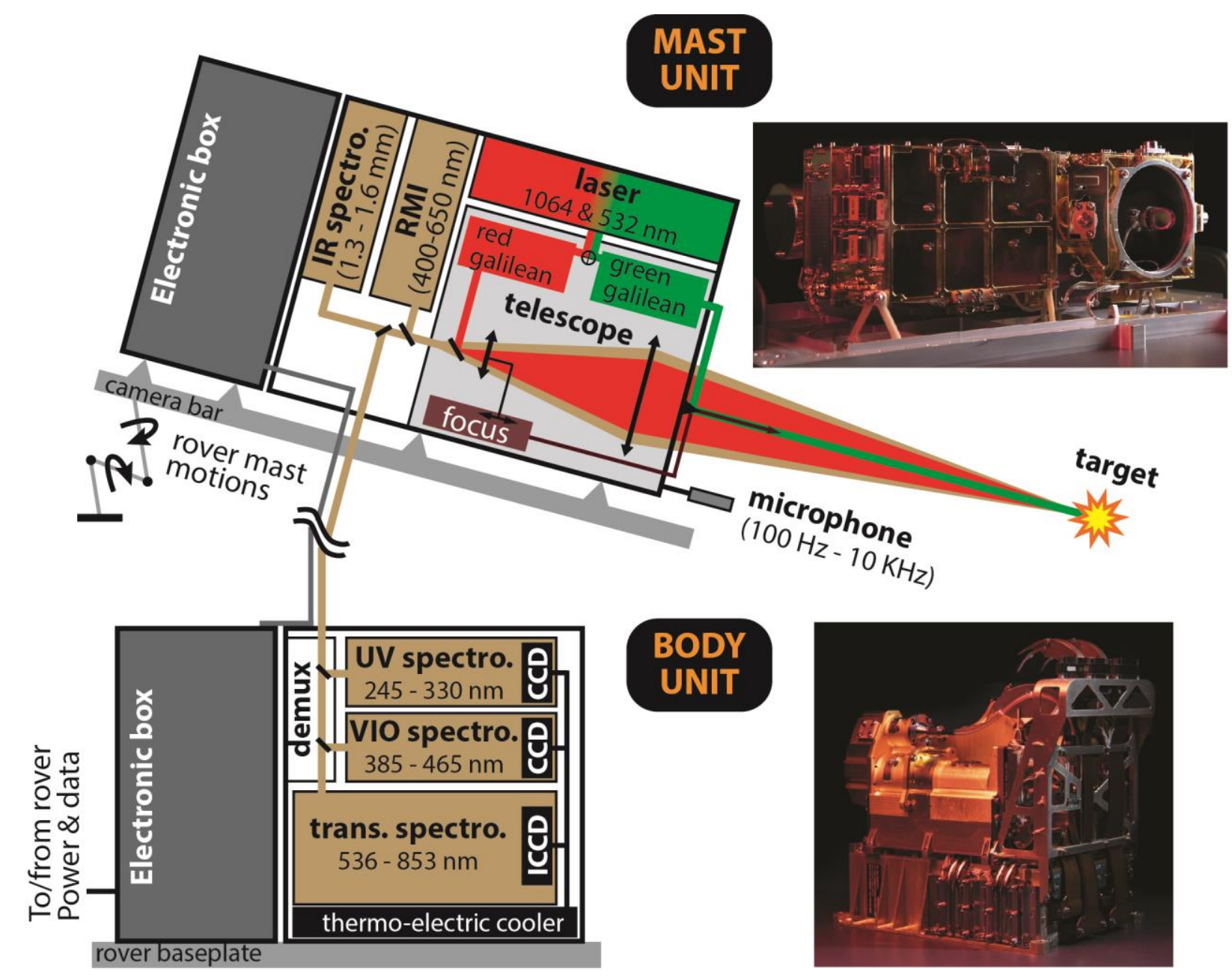

Figure 4 